OPEN ACCESS

Edited by:

Shemin Lu,

Xi'an Jiaotong University, China

Reviewed by:

$\mathrm{Nan} \mathrm{Mu}$,

Fourth Military Medical University,

China

Rowan Hardy,

University of Birmingham,

United Kingdom

*Correspondence:

Caihong Wang

snwch@sina.com

Specialty section:

This article was submitted to

Autoimmune and

Autoinflammatory Disorders,

a section of the journal

Frontiers in Immunology

Received: 13 May 2021

Accepted: 01 July 2021

Published: 19 July 2021

Citation:

Cheng $L$, Wang $Y$, Wu $R$,

Ding T, Xue H, Gao C, Li X and Wang C (2021) New Insights From

Single-Cell Sequencing Data:

Synovial Fibroblasts and Synovial Macrophages in Rheumatoid Arthritis.

Front. Immunol. 12:709178.

doi: 10.3389/fimmu.2021.709178

\section{New Insights From Single-Cell Sequencing Data: Synovial Fibroblasts and Synovial Macrophages in Rheumatoid Arthritis}

\author{
Liyun Cheng ${ }^{1}$, Yanyan Wang ${ }^{1}$, Ruihe $W^{1}{ }^{1}$, Tingting Ding ${ }^{1}$, Hongwei Xue ${ }^{1}$, Chong Gao ${ }^{2}$, \\ Xiaofeng $\mathrm{Li}^{1}$ and Caihong Wang ${ }^{1 *}$ \\ ${ }^{1}$ Department of Rheumatology, the Second Hospital of Shanxi Medical University, Taiyuan, China, 2 Pathology, Joint \\ Program in Transfusion Medicine, Brigham and Women's Hospital/Children's Hospital, Harvard Medical School, Boston, \\ MA, United States
}

Single-cell RNA sequencing (scRNA-seq) technology can analyze the transcriptome expression level of cells with high-throughput from the single cell level, fully show the heterogeneity of cells, and provide a new way for the study of multicellular biological heterogeneity. Synovitis is the pathological basis of rheumatoid arthritis (RA). Synovial fibroblasts (SFs) and synovial macrophages are the core target cells of RA, which results in the destruction of articular cartilage, as well as bone. Recent scRNA-seq technology has made breakthroughs in the differentiation and development of two types of synovial cells, identification of subsets, functional analysis, and new therapeutic targets, which will bring remarkable changes in $\mathrm{RA}$ treatment.

Keywords: single-cell RNA sequencing, rheumatoid arthritis, synovial fibroblast, synovial macrophage, biomarkers

\section{INTRODUCTION}

Rheumatoid arthritis (RA) is a progressive aggressive immune disorder which can lead to increased mobility and disability, the main features of this disease are persistent synovitis, pannus formation, joint destruction, and adjacent bone erosions (1-3). At present, it is considered that environmental and genetic factors are related to the pathogenesis of RA, its etiology and pathogenesis have not been fully elucidated and remain to be clarified (4). The traditional therapeutic approaches of RA are suppressing the excessive immunological response and inflammatory reaction, which can only help to relieve RA symptoms and delay disease progression rather than cure (5). In addition, these strategies lead to several systemic side effects (6). Therefore, to explore the effective molecular targets for the treatment of RA is a focus of research (7).

ScRNA-seq is a technology for high-throughput sequencing and analysis of genome and transcriptome at single-cell level. It finds rare new cell subpopulations and shows the changes in each cell. It is a new technology to study the genetic heterogeneity of multicellular organisms which plays increasingly remarkable role in cancer research, developmental biology and neuroscience (8-10). With regards to rapid development of ScRNA-seq, the development lineage of immune cells is revealed in the field of autoimmune diseases, and the gene modules and regulatory procedures that determine the immune response are identified $(11,12)$. The latest research has reported the 
heterogeneity of synovial fibroblasts and macrophages in RA and the differences of their origins and biological characteristics. The purpose of this paper is to further clarify the pathogenesis of RA from the single cell biological level and explore new therapeutic breakthroughs.

Whether the tumor like abnormal proliferation and erosiveness of RA synovial fibroblasts are related to their heterogeneity has always been the focus of scholars' research. it is well known that the different functions occur of fibroblasts in different anatomical site (13). However, there is no unified and comprehensive classification of synovial fibroblasts, and the specific markers of different subpopulations, and their specific roles in the pathological process of RA have not been fully elucidated. Single cell sequencing can maximize the genetic information of a single cell and discover the heterogeneity between cells. According to different algorithms, cells with different commonness are divided into different subpopulations, and compared with the known library to identify specific cell markers of different communities, and further discover and identify new cell surface markers. Compared with synovial fibroblasts, the action of synovial macrophages in the pathophysiology and pathological processes of RA is poorly understood. Because the number of synovial macrophages is limited and difficult to obtain, the origination and exact function of synovial macrophages in inflammatory diseases are not fully known (14).With the development of ScRNA-seq in recent years, the research of synovial fibroblasts has stepped to a new level. ScRNA-seq can study the development spectrum of synovial fibroblasts and macrophages on the basis of single cell, and dynamically analyze cell heterogeneity. It plays an imperative role in finding new targets for RA treatment, and has become a significant research approach. The core steps of ScRNA-seq technology and its application in the research of synovial fibroblasts and macrophages in RA will be introduced in this paper.

\section{SINGLE CELL SEQUENCING TECHNOLOGY}

The development and application of next-generation sequencing technology have brought great changes to the development of biological research. However, the results obtained by traditional population sequencing are the average value of the whole sample, or reflect the data of the dominant number of cells. In 2009, Tang et al. (15) achieved the first single cell transcriptome sequencing by improving the previously used single cell transcriptome amplification method for microarray analysis. With the development of sequencing, cell separation and genome-wide amplification, single cell sequencing technology has been improved and is becoming the focus of life science research.

Single-cell sequencing (SCS) has mushroomed as a powerful novel set of technologies in NGS, consisting of single-cell DNA sequencing, scRNA-seq, along with single-cell epigenomic sequencing (16). Among them, scRNA-seq, which is the most widely used, can reveal the subtle changes of transcriptome of each cell, clarify the heterogeneity of mRNA expression among individual cells, and obtain more sample information by highly efficient amplification and high-throughput sequencing. At the same time, scRNA-seq technology can solve the problem of low sample acquisition. Hundreds to thousands of cells can meet the needs of sequencing. The sample size is flexible. It plays an indispensable role in revealing the source and function of cells, finding new functional cell subsets and therapeutic targets.

ScRNA-seq is used to amplify and sequence mRNA at a single cell level through high-throughput detection. Generally, it includes the following steps: (1) isolation and cleavage of single cell or single cell nucleus; (2) reverse transcription; (3) cDNA amplification; (4) construction of sequencing library (17) (Figure 1). The separation and capture of single cell and reverse transcription and amplification of cDNA with minimally mRNA are the two key points in the whole technical process. The development of ScRNA-seq technology largely depends on the

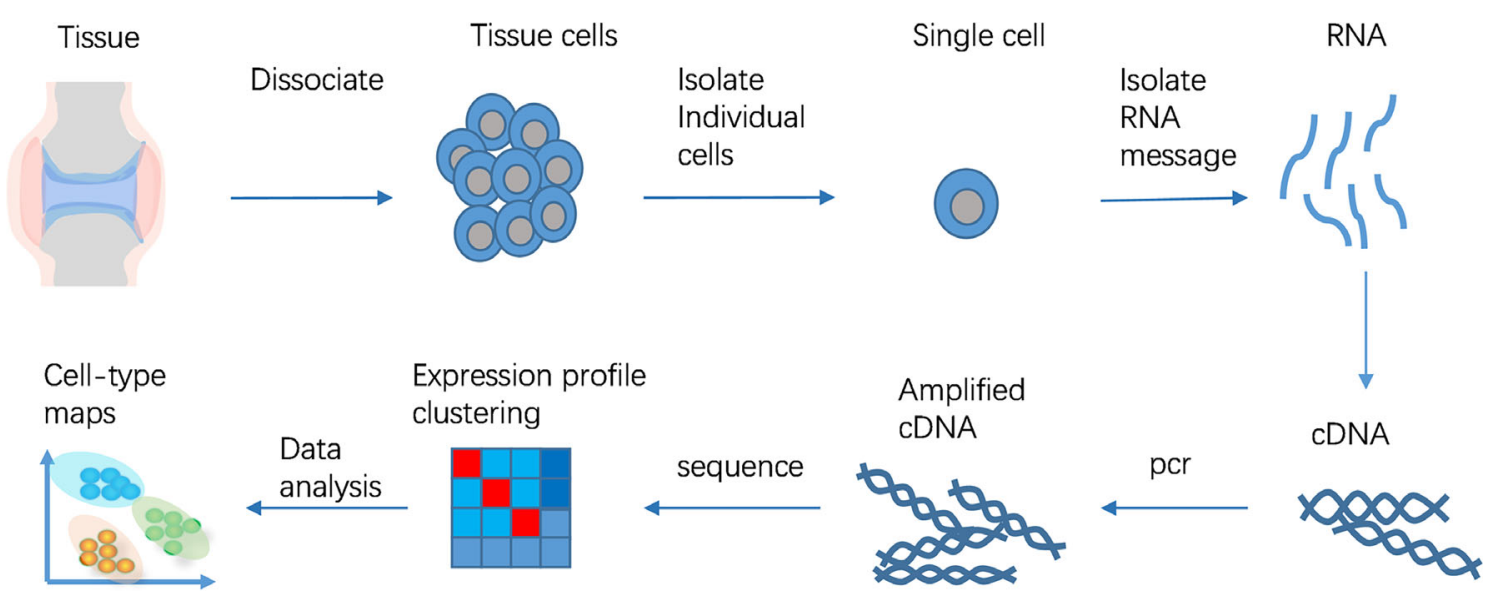

FIGURE 1 | ScRNA-seq workflow. The ScRNA-seq process includes isolation and cleavage of single cell or single cell nucleus, reverse transcription, cDNA amplification, construction of sequencing library. 
continuous optimization of the solutions to these two problems. The frequently utilized single cell separation approaches include continuous dilution, magnetic activated cell sorting, micromanipulation, fluorescence activated cell sorting, microfluidic platform, and laser capture microdissection (18). Because of the small sample size of ScRNA-seq technology, the optimization of experimental process and the accuracy of steps are of great significance. Different cell capture methods, cDNA amplification and library construction methods are being reported and used. New ScRNA-seq platform is also constantly improving. ScRNA-seq technology is developing towards the direction of gradually reducing the cost and increasing the throughput.

\section{SCRNA-SEQ OF SYNOVIAL FIBROBLASTS}

\section{Function of Synovial Fibroblasts}

In health the joint synovium is a delicate and thin structure, which encapsulates articular joints and functions as a homeostatic balance of the synovial fluid for efficient and smooth movement. The structure of synovium can be divided into two layers: the sublining, as well as lining layer of which in a healthy joint constitutes of one to two cell layers thick. There is no obvious boundary between the two layers. The synovial intimal lining directly interfaces with the synovial fluid and is composed of spindle-shaped fibroblasts aligned in a cohesive layer. Macrophages are distributed in the fibroblast layer. This layer enhances the barrier role, as well as secretes hyaluronic acid and lubricin. Less densely packed fibroblasts along with macrophages in a loose tissue matrix coupled with a network of blood vessels make up the sublining layer (19).

The synovial lining layer goes through remarkable hyperplasia, occasionally reaching a depth of 10-15 cells in RA. The compartment of synovial fibroblast expands in extensive numbers, resulting in remodeling of tissues with formation of papilla. At the same time, synovial fibroblast experiences epigenetic alterations, assuming a stable and activated phenotype, with the ability of infiltrating damaged articular cartilage, as well as the bone $(20,21)$. The sublining layer likewise expands, with inflammatory cell infiltrates, consisting of macrophages, plasma cells, T cells along with B cells (22). Synovial fibroblasts and macrophages play an indispensable role in joint destruction and disease persistence (23-25). Synovial fibroblasts contribute to disease progression by producing disease-linked cytokines, chemokines, as well as extracellular matrix remodeling components $(26,27)$ (Figure 2).

In RA, synovial fibroblasts can promote inflammation and cartilage destruction, while the interaction between $\mathrm{T}$ cells and synovial fibroblasts promotes $\mathrm{T}$ cell recruitment and synovial fibroblasts activation (28). The inflammatory cells produced TNF-a to induces vascular endothelial cells and synovial cells to produce CXCL13, which recruits circulating B cells and T cells to the inflammatory site and forms ectopic germinal center.

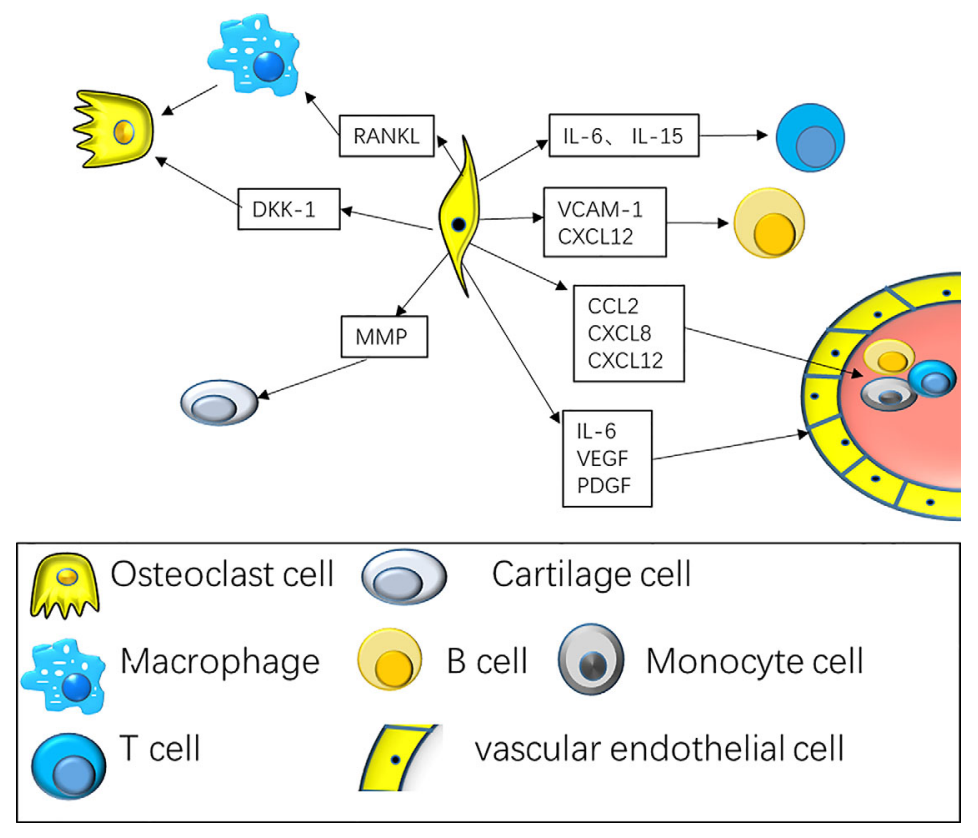

FIGURE 2 | Synovial fibroblasts interact with various types of immune cells to maintain synovitis and continued bone destruction. The interaction of fibroblasts with T cells along with $\mathrm{B}$ cells includes the secretion of cytokines consisting of CXCL8, CCL2, CCL5, to promote the mobilization and retention of T cells and B cells. At the same time, fibroblasts cytokines consisting of IL-6 and IL-15 are specifically remarkable for the differentiation of Th17 cell subsets; Fibroblasts promote angiogenesis by secreting VEGF, PDGF. Fibroblasts secrete RANK Ligand, that enhances osteoclast differentiation along with activation resulting in bone erosion, and DKK-1 that represses anabolic osteoblast function, averting repair of bone erosions. 
When joint derived helper $\mathrm{T}$ cell 17 (Th17) is activated and recruited into the joint to induce inflammatory response, synovial fibroblasts can secrete CCL20 in response to IL-17 and other pro-inflammatory cytokines, and accelerate Th17 cell recruitment to induce and enhance arthritis (29). Studies have shown that there were specific staining of CD4, CXCR5 and ICOS in RA synovial tissue, suggesting that follicular helper T cell (Tfh) cells may exist in synovial tissue. Studies on co-culture of synovial fibroblasts and Tfh cells show that RA synovial fibroblasts can promote the proliferation of peripheral blood Tfh cells by secreting IL-6 (28). IL-21 is mainly secreted by Th17, Tfh and natural killer T cell (NKT). The proliferation of RA-FLS was up-regulated in the presence of IL-21. Tfh cells may promote the proliferation of synovial fibroblasts by secreting IL-21.

Fibroblasts recruit and retain $\mathrm{B}$ cells by secreting chemokines (such as CXCL12, CXCL13, VCAM1) and interacting with cell surface adhesion (30). Meanwhile, they secrete BAFF and April to maintain the survival and differentiation of B cells (31). The signal between FLS and B cells is bidirectional. RA-FLS also contributes to the differentiation and activation of B cells, and then B cells can produce a variety of autoantibodies. At the same time, B cells can stimulate RA-FLS to produce more IL-6. Recently, it was show that, the expression of RASF proliferation inducing ligand and B cell activating factor mediated by TLR mediate the survival and function of B cells in RA synovium (32).

It is generally believed that the activation of RA-FLS stimulates peripheral blood monocytes to enter the articular cavity and differentiate into macrophages through paracrine effect (33). In rheumatoid arthritis synovial fluid, macrophages form the largest population of immune cells in rheumatoid arthritis and play a role in synovitis by producing cytokines such as TNF, IL-1 and IL- 6 and chemokines such as CCL2 and CXCL8 (34). Alivernini et al (35). shows MerTK ${ }^{\text {pos }}$ CD206 ${ }^{\text {pos }}$ STMs cluster can induce the inflammatory response of synovial fibroblasts and promote synovial inflammation by producing pro-inflammatory cytokines and alarm proteins. MerTK ${ }^{\text {neg }} C D 206^{\text {neg }} S T M$ cluster can produce lipid mediators, induce FLS repair response and alleviate synovial inflammation. FLS can promote the production of RANKL by macrophages and promote osteoclast. The invasion of rheumatoid arthritis synovial fibroblasts is related to the stimulation of IL-1 $\beta$ and the inflammatory response of macrophages. Hypoxia makes RAFLS secrete more TGF- $\beta$ and promote macrophages to polarize to M2 and anti-inflammatory factor to play a down-regulation role (36).

\section{Identified New Synovial Fibroblasts Subsets and Surface Markers}

Synovial fibroblasts are the core target cells of a remarkable immune effect in synovial tissue. They interact with lymphocytes, macrophages, as well as other immune cells in the synovium by generating pro-inflammatory cytokines, chemokines along with tissue-destructive factors consisting of IL-6, IL-8 and MMPs (27, 37-39) and play an imperative role in the continuous inflammation and bone destruction of RA synovium $(40,41)$. Traditional research methods have shown that synovial fibroblasts have certain heterogeneity. Synovial fibroblasts isolated from diverse joints and the same joint lining layer and sublining layer show distinct phenotypes and they are different in their gene expression trends, epigenetic marks and function (42-44). Synovial fibroblasts with different phenotypes have distinct characteristics of adhesion, proliferation, chemotaxis and matrix degradation, as well as different responses to TNF, thus forming a unique microenvironment in each joint $(44,45)$. At present, a variety of fibroblast surface markers are known, the lining layer fibroblasts are identified via the expression of a cell surface biomarker termed as cadherin-11, which allows homotypic adhesion of the lining layer fibroblasts to one another to facilitate the generation of a functional lining layer in the absence of a basal lamina $(46,47)$. Other biomarkers have also been linked to the lining layer fibroblasts, for instance adhesion molecule VCAM-1, CD55, FAP (fibroblast activation protein), and podoplanin (GP38) (48-50). Sublining fibroblasts are identified with alternative biomarkers, for instance CD90 (THY1) or CD248 (endosialin) and seem to have diverse roles to lining layer fibroblasts $(49,51,52)$. With the continuous development of experimental technology, new subsets of synovial fibroblasts are being discovered, and the functions of different subsets are also constantly defined.

Recently, the independent subsets of RA synovial fibroblasts were identified by scRNA-seq technology. There are significant differences in the anatomical position, transcriptome differences and functions of these subpopulations in synovial tissue.

Stephenson et al (53). sequenced 20387 single cells from synovial tissue of 5 patients with rheumatoid arthritis, and found three different subpopulations of RA synovial fibroblasts, which can be divided into two groups according to different surface markers, one is CD55+ synovial fibroblast, the other is CD90+ subset. CD55 is a glycosylphosphatidylinositol-anchored complement-modulating protein (decay-accelerating factor), expressed by synovial fibroblasts with high local abundance in the intimal lining layer, which can protect synovium from immune complex mediated arthritis (48). CD55+ fibroblasts locate to the intimal lining and are responsible for synovial fluid formation and turnover. Of note, hyaluronan synthase 1 (HAS1) (53, 54), lubricant PRG4 (55) and DNASE1L3 were highly expressed. Previous studies have shown that lubricin/ proteomeglycan-4 (PRG4) is a mucus glycoprotein secreted by synovial fibroblasts and superficial chondrocytes, which has a variety of homeostasis effects in the joint and play an antiinflammatory role by combining with TLR2 and TLR4 (56). HAS is mainly divided into three subtypes: HAS1, HAS2 and HAS3, and the three subtypes are independent and differentially regulated, play a different role in arthritis. Among them, TGF- $\beta$ upregulates HAS1 mRNA $(57,58)$. Furthermore, Go enrichment analysis indicated that CD55+ fibroblasts expressed functional modules linked to endothelial cell proliferation and modulation of reactive oxygen species responses (53). CD90+ fibroblasts are mainly located in the lower layer of synovial sublining layer, which are enriched for modules linked to metallopeptidase activity, as well as the organization of the extracellular matrix (53). 
Mizoguchi et al. (59) identify seven different fibroblast surface protein phenotypes and classified them into three subsets according to the expression of podoplanin (PDPN) $(60,61)$, cadherin-11 (CDH11) (46, 62), THY1 (also known as CD90) (63) and CD34 (64) by integrating transcriptomic data. they found that different subsets of fibroblasts play different roles in joint inflammation and bone destruction. CD34, a remarkable marker of stem/progenitor cells, is an intercellular adhesion molecule. It is expressed in some synovial fibroblasts (65), and also in endothelial cells and nerve tissues (66). CD34+ fibroblasts were reported in superficial lining, as well as deeper sublining synovium areas. They proliferated actively in the synovium of RA. At the same time, they promoted the progress of joint inflammation by secreting a large number of inflammatory factors and mediating the enrichment of leukocytes, which was related to the migration of fibroblasts (59). CD34-THY1fibroblasts were remarkably reported in lining area and express BMP-6, thought to enhance formation of osteoblastic bone (59, 67). in RA patients the count of CD34-THY1+ fibroblasts is threefold than that in OA, they generate a discrete perivascular zone, which surrounds the capillary structures in the synovium deep sublining layer, especially near aggregations of lymphocytes, and play an indispensable role in matrix infiltration, immune cell mobilization and osteoclast formation. At the same time, through its overexpression of TNFSF11 [also referred to as receptor activator of nuclear factor- $\kappa \mathrm{B}$ ligand (RANKL)] $(68,69)$, CD34-THY1+ fibroblasts participate in the transport of $\mathrm{T}$ cells in autoimmune inflammation. Therefore, this subgroup may also be responsible for RA lymphocyte accumulation in synovium (59). Transcriptome sequencing and scRNA-seq have the same conclusion, which indicates that gene differences reflect biological differences rather than technical or random differences (59) (Figure 3).

In the larger study, the synovial tissues of $51 \mathrm{RA}$ or OA patients were used for single-cell sequencing and transcriptomics to analyze the cell subsets associated with arthritis (55). Among 1844 synovial fibroblasts, four presumed subpopulations were identified: CD34+ fibroblasts, HLA-DRAhi fibroblasts, and Dkk3 + fibroblasts were located in the sublining layer, while CD55+ fibroblasts were located in the lining layer (55). HLADRAhi fibroblasts highly express CXCL12 and HLA-DRA, and is the main source of IL-6. IL-6 is mainly secreted from proinflammatory M1 macrophages along with RA synovial tissue fibroblasts $(70,71)$. Along with IL-1 $\beta$, IL-6 has an imperative

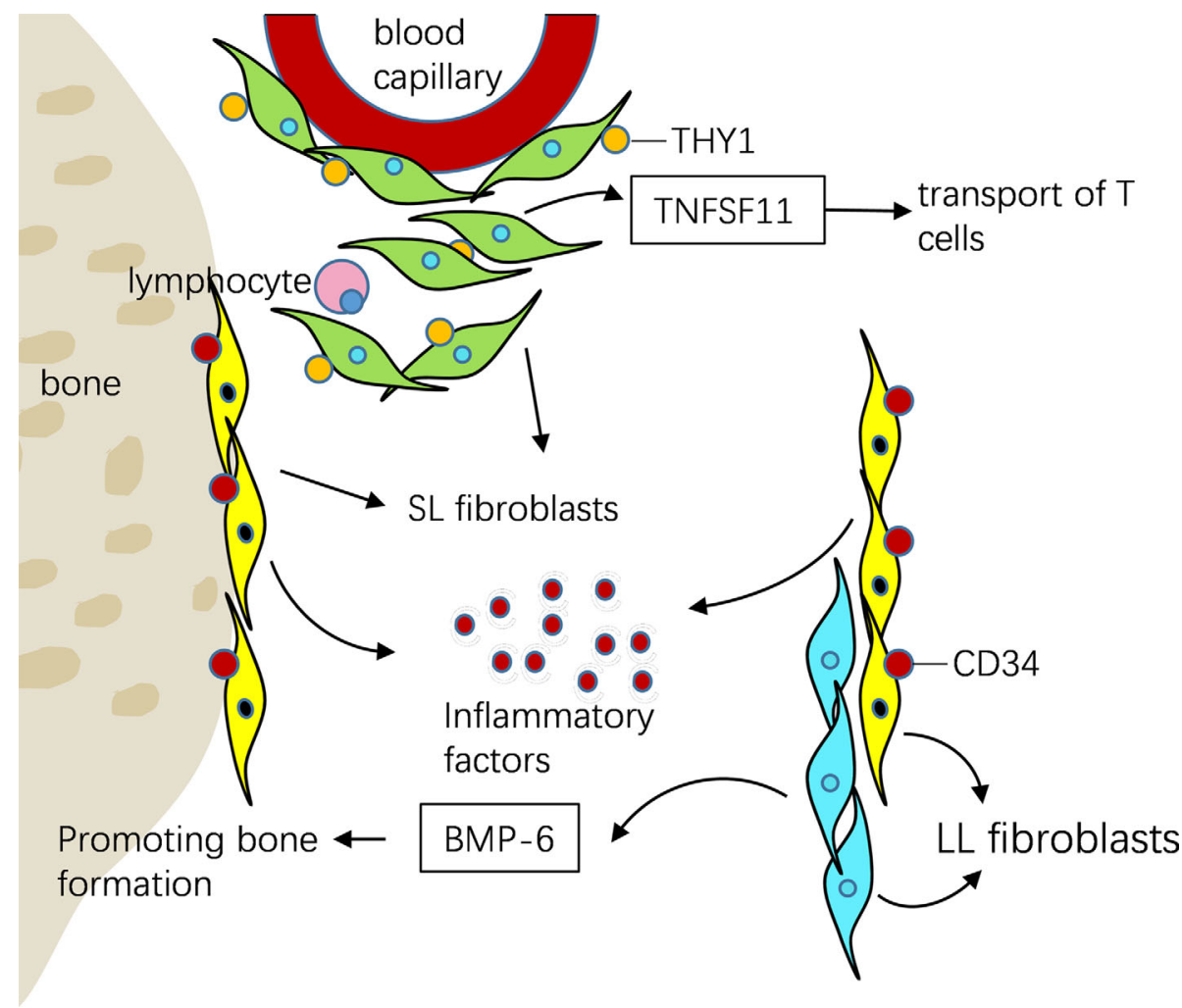

FIGURE 3 | ScRNA-seq showed that three different fibroblasts were distributed in different parts of synovial tissue and play different roles in RA. CD34+ fibroblasts were reported in superficial lining, as well as deeper sublining areas of the synovium. They promoted the progress of joint inflammation by secreting a large number of inflammatory factors. CD34-THY1- fibroblasts were remarkably reported in lining area and express BMP-6, recognized to enhance osteoblastic bone formation. CD34-THY1+ fibroblasts in RA produce a discrete perivascular zone that surrounds capillary structures in the deep sublining layer of the synovium, it participate in the transport of T cells by overexpression of TNFSF11. 
role for Th17 cell differentiation, which is remarkable in RA angiogenesis (72-75). At the same time, IL-6 can activate Tfh by up regulating signal transducer and activator of transcription (STAT-1 or STAT-3), depending on the expression of bcl-6 (76). The antagonists of TNF-a and IL-1B can down regulate the number of Tfh cells by reducing the level of IL-6 (28). The RA fibroblasts stimulated by adiponectin can promote the production of TFH cells by producing IL-6. Intra articular injection of ad aggravates synovial inflammation and increases the frequency of Tfh cells in CIA mice (77). HLA-DR is a class of MHC-II, which is considered to present exogenous antigen. HLA-DR and CD68 are co-expressed in Macrophage-like synoviocytes (78). DKK3+ fibroblasts was a novel subtype of sublining fibroblast, characterized by elevated expression of DKK3, CADM1, and COL8A2, and can prevent cartilage degradation in vitro $(55,79)$. These subsets may be the key inflammatory subsets of RA.

\section{To reveal the New Function of Different Subsets of Synovial Fibroblasts}

Chronically activated fibroblasts account for the degradation of excessive matrix, destroying cartilage, resulting in permanent joint damage in RA by activates osteoclasts (80). Targeted treatment of synovial fibroblasts may become an effective strategy for the treatment of RA. Recent studies have shown that Notch signaling plays a role in THY1 expressing perivascular and subcutaneous fibroblasts, and blocking Notch3 signaling can alleviate joint inflammation in mice (81). Another study showed, specific small molecule agonist can activate melanocortin type 1 receptor (MC11), make synovial fibroblasts activate GPCR and aging, so as to promote the regression of arthritis (82).

Mizoguchi et al. (59) found that different fibroblast subpopulations play different roles in joint inflammation and bone destruction.CD34-THY1+ fibroblasts are abundant around blood vessels in RA synovium, with their expression profile revealing prospective pathogenic functions in matrix infiltration, immune cell mobilization, and osteoclastogenesis. CD34-THY1+ fibroblasts are located around the vessels under the synovial lining, which are related to the activity of disease, the infiltration of immune cells and the increase of bone destruction. In RA, a remarkable pathogenic effector role of synovial fibroblasts is modulation of osteoclastogenesis, a process dominantly carried out by TNFSF11 and opposed by TNFRSF11B [also referred to as osteoprotegerin (OPG)], which is a decoy receptor for TNFSF11. An exploration of the genes linked to osteoclastogenesis exhibited high expression of TNFSF11, but low TNFRSF11B expression in CD34-THY1+ fibroblasts.

FAP $\alpha$, a cell-membrane dipeptidyl peptidase (83), was remarkably higher in synovial tissue along with cultured synovial fibroblasts isolated from individuals who fulfilled classification criteria for RA in contrast with patients in whom joint inflammation resolved, implying that FAP $\alpha$ expression might be linked to a pathogenic fibroblast phenotype $(84,85)$. A new study in 2019 (2) showed that FAP $\alpha$ was expressed in the sub-lining and lining layer fibroblasts. According to the expression of THY1, synovium can be divided into FAP $\alpha+$ THY $1+$ lining layer fibroblast and FAP $\alpha+$ THY $1-$ sublining fibroblast. Five subtypes of fibroblasts were identified by single cell sequencing of inflammatory joints in RA mice, and similar results were obtained in synovium of RA patients. Further research shows that, FAP $\alpha+$ THY $1+$ fibroblasts mediate synovial inflammation by secreting cytokines and chemokines, while FAP $\alpha+$ THY 1- plays a role in bone destruction by expressing osteoclast activity inducers consisting of matrix metalloproteinases, suggesting that synovial fibroblasts at different anatomical positions play different roles in the pathogenesis of RA.

\section{Dynamic Changes of Synovial Fibroblast Subsets in Inflammatory State}

The degree of synovial cells and immune cells infiltrate is a highly variable phenomenon in different disease stages and drug exposure in RA (86-88), the degree of synovitis is related to the clinical phenotype of RA and as such has been explored as a prospective source of predictive, as well as prognostic biomarkers in RA (88). Recent research shows that different subsets of synovial fibroblasts play an independent role in the process of disease. Therefore, scholars speculate that the proportion of fibroblast subpopulations related to disease may be different in different diseases and different stages of RA. ScRNA-seq also showed the dynamic changes of synovial fibroblast subsets in inflammatory state.

Mizoguchi et al. (59) showed that there was a significant difference in the proportion of the three synovial fibroblast subsets between OA and RA synovium, CD34-THY1+ fibroblasts accounted for $22 \%$ of the total fibroblasts in RA and $8 \%$ in OA. There were less CD34-THY1-, more CD34-THY1+ and more $\mathrm{CD} 34+$ fibroblasts in swollen joints. In addition, the proportion of CD34-THY1+ fibroblasts was correlated with the proportion of leukocyte infiltration, synovitis and synovial hypertrophy, which indicated that the altered proportion of fibroblast subsets in RA reflected the molecular level and clinical level of tissue inflammation. Huang et al. (89) showed that some common fibroblast markers, consisting of procollagen I (COL1A1), Prolyl-4-hydroxylase (P-4-H), Vimentin, along with procollagen III (COL3A1), are different in OA FLS and RA FLS. Besides, RA FLS exhibits more severe cellular behavior in contrast with OA FLS, entailing a more rapid rate of proliferation, stronger invasive potential, and elevated expression as well as secretion of inflammatory cytokines. Additionally, elevated expression of inflammatory markers, consisting of CCL2, IL- 6 , IL-1 $\beta$ and TNF- $\alpha$, were also reported in RA FLS in contrast with FLS isolated from the less inflamed OA synovium. This suggests that different subsets of fibroblasts may play a role in the pathogenesis of OA and RA.

The continuous progress of ScRNA-seq technology has opened the era of exploring new targeted drugs for synovial fibroblasts. At present, there are no specific drugs targeting synovial fibroblasts. Therefore, identifying the heterogeneity of fibroblasts and pathogenic fibroblast subsets to determine the disease-related fibroblast subsets that can be used as specific 
targets for disease treatment may provide new effective strategies for treating RA $(90,91)$. Single cell sequencing of synovial fibroblasts (55) will also serve as a research template to identify pathogenic interstitial fibroblast subsets in other autoimmune diseases, for instance connective tissue disease related pulmonary interstitial disease.

\section{SINGLE-CELL RNA-SEQUENCING OF SYNOVIAL MACROPHAGE}

Previous studies have shown that the aggregation of monocyte macrophages can promote the occurrence and development of arthritis, and the infiltration of synovial macrophages is positively correlated with the progress of joint destruction (25, 92). With the development of research technology, the research on the heterogeneity of synovial macrophages is also in-depth. ScRNA-seq leads to different understanding of synovial macrophages, which have heterogeneous subpopulations with different sources and biological characteristics.

\section{Origin and Biological Heterogeneity of Synovial Macrophage Subsets}

For numerous years, it was speculated that macrophages primarily originated from circulating monocytes differentiation, however recently morphological along with functional differences between these cells dispute this speculation $(93,94)$. Nonetheless, a series of recent reports have documented that the origins of macrophages in different tissues/organs are not exclusively originated from circulating monocytes. Tissue-resident macrophages derive mainly from embryonic progenitors and to less degree from intermediates of circulating monocytes, additionally many of them are capable of self-renewal (95-98). The mobilized population of monocyte originated macrophages significantly increase during inflammatory conditions. It is generally believed that RA-FLS can stimulate peripheral blood monocytes to enter articular cavity and differentiate into macrophages through paracrine effect after activation (33). By the different transcriptional and epigenetic characteristics showed in macrophages different subsets, the unique functional patterns of variant tissue were indicated. It was illustrated that embryonic macrophages participate in tissue remodeling, while adult-originated macrophages mainly assist in host defense.

Macrophages constitute remarkable plastic cells, with the ability switch from one phenotype to another (99). Synovial macrophages can be divided into different specific subsets according to their origins and functions. Two primary macrophage subpopulations with different roles consist of classically activated or inflammatory (M1) and alternatively activated or anti- inflammatory (M2) macrophages have been recognized (100). M1 macrophages that mediate resistance to pathogens and tissue destruction by produce pro- inflammatory cytokines like TNF, IL-6 and IL-1 $\beta$, CCL2, IL-8, IL-12 and IL-23 (101, 102); M2 macrophages can remove debris and promote tissue repair by produce anti-inflammatory cytokines consisting of TGF- $\beta$, IL-10, IL-4, IL-13 (103). In RA, there is a positive correlation between the infiltration of synovial macrophages and the progress of joint destruction (92). The imbalance of M1/M2 in synovium is one of the remarkable reasons for chronic synovitis. Misharin et al. (104) showed that macrophages can be transformed from $\mathrm{M} 1$ to $\mathrm{M} 2$ in the progression of RA to promote damage repair. After co-culture of RA-FLS and macrophages in normoxia, the cytokines secreted by RA-FLS can strongly inhibit the pro-inflammatory activity of M1 and enhance the expression of genes promoting M2 polarization (105). Deciphering the process of macrophage polarization, mobilization, and functions may provide insights for the development of new therapies for RA. At the same time, Zhang et al. (106) showed that Macrophages with M1 polarization gather in synovium of OA patients and OA model mice. M1 polarization of macrophages promotes synovium hyperplasia, synovitis and progression of OA. Controlling the polarization of synovium macrophages to M2 may be a new strategy for prevention and treatment of OA.

In the latest study (107), Two types of SM were found SM after tracking macrophages in mice: embryonic SMs (ESMs), and bone marrow-derived SMs (BMSMs). ESM expressed antiinflammatory cytokines, consisting of IL-4 and IL-10, while BMSM expressed pro-inflammatory cytokines, consisting of IL- $1 \beta$ and TNF. In arthritic mice, the number of ESMS reduced during disease development and then increased during regression, whereas BMSM was the opposite. This study first confirmed that synovial macrophages have at least two origins, ESM and BMSM, and their effects are different. Secondly, this study also showed that two types of SM also exist in the synovium of RA patients and have similar anti-inflammatory and anti-inflammatory phenotypes of ESM and ESM. Through the different cell characteristics and dynamic expression patterns in RA patients/CIA mice, it was revealed that two subpopulations of different origins, embryonic ESM (anti-inflammatory) and bone marrow derived BMSM (pro-inflammatory), play different roles in arthritis.

In Monocyte of human RA synovium, IL1B+ proinflammatory monocytes (SC-M1), NUPR1+ monocytes (SC-M2), C1QA+ monocytes (SC-M3), and interferon (IFN) activated monocytes (SC-M4) were identified by Single cell sequencing. Among them, SC-M3 and SC-M4 matched with those of mouse resident synovial macrophages, while SC-M1 and SC-M4 were similar to those of mouse monocyte derived synovial macrophages (55).

To elucidate the immunomodulatory mechanism of drug-free remission in RA, Alivernini et al. (35) analyzed 32000 STMs of patients with early/active RA, refractory/active RA, and RA in persistent remission using single-cell transcriptional analysis to identify the phenotypic changes. MerTK ${ }^{\text {pos }} \mathrm{CD} 206^{\text {pos }}$ and MerTK ${ }^{\text {neg }} C D 206^{\text {neg }}$ STM contain nine different clusters, which can be divided into four subgroups with different functions of homeostasis, regulation and inflammation. Compared with the healthy control group, the number of MerTK ${ }^{\text {pos }} \mathrm{CD} 206^{\text {pos }}$ increased in remission stage and $\mathrm{MerTK}^{\text {pos }} \mathrm{CD}_{206^{\text {pos }}}$ cluster 
increased in active stage. Therefore, the tissue residence of MerTK $^{\text {pos }}$ CD206 $^{\text {pos }}$ STM seems to play an important role in maintaining the sustained remission of inflammation. The decrease of MerTK ${ }^{\text {pos }}$ STM proportion in remission stage is related to the increased risk of disease after drug withdrawal. Therefore, the regulation of mertkpos STM may be a potential treatment for RA. Other studies have shown that the subsets of HBEGF+ inflammatory macrophages were enriched in RA tissue and formed by resident fibroblasts and cytokine tumor necrosis factor (TNF). Other studies have shown that the subsets of HBEGF+ inflammatory macrophages are enriched in RA tissue and contribute to fibroblast mediated joint destruction (108).

\section{Synovial Macrophages Created a Protective Barrier for the Joint}

ScRNA-seq can map a single cell of a given lineage to a unified orbit, so as to clarify the time sequence of cell development and differentiation and infer the development trajectory of cells from a new perspective by using this cross-time continuum. Known as "pseudotime" (109).

In 2019, Gerhard et al. (110) first discovered a new subpopulation of synovial macrophages, which serves as a protective and tightly connected barrier of synovial macrophages. The researchers used a variety of methods, including single cell sequencing, to study the subsets and functions of macrophages in different states. CX3CR1 is a kind of chemokine receptor that can be used by monocytes, CX3CR1+ lining macrophages and CX3CR1- interstitial macrophages are two kinds of tissue-resident macrophages origin from the embryo, among them, CX3CR1- stromal synovial macrophages can be divided into specific subsets according to the expression of AQP1, MHCII and RELM- $\alpha$. Both RELM $\alpha+$ macrophages and CX3CR1+ lining macrophages are derived from proliferative MHCII+ interstitial macrophages (110). This study found that CX3CR1+ tissue resident macrophages derived from CX3CR1 monocytes form an internal immune barrier in synovial lining and physically isolate joints (110).

Using an arthritis mouse model in which macrophages might be tracked via engineering them to be fluorescent, the authors reported that the barrier layer was remarkably dynamic. Upon inducing arthritis, the layer experienced active remodeling causing loosening of the physical cross talks between lininglayer fibroblasts and barrier macrophages. The barrier macrophages might ingest and remove inflammatory immune cells called neutrophils that aggregate and die in the synovial fluid in arthritis. These CX3CR1+ macrophages in the lining layer of synovium provide tight junction mediated shielding for intra-articular structures, forming a protective macrophage barrier and restricting inflammatory response.

When comparing the single-cell RNA data from mice with similar data sets available from an assessment of the joints of individuals with $\mathrm{RA}$, the gene-expression trends of the macrophage subsets matched up between the two species. This implies that cells, which are similar to the barrier, as well as interstitial macrophages in mice could similarly exist in humans, and hence be relevant to human disease. This study reveals the unexpected functional diversity among the synovial macrophages, and the new subpopulation of the protective barrier effect of the synovial macrophages, which is of new significance for the study of the heterogeneity of macrophages in health and disease.

\section{REVIEWS AND PERSPECTIVES}

The continuous application of ScRNA-seq technology can help us to identify the heterogeneity of cells so as to determine the targets related to the treatment of RA, and also play an important role in tracking the lineage or development relationship between synovial cells and immune cells, osteoclasts and other cells, so as to further discover the synovial fibroblasts, synovial macrophages and other immune cells in RA and making the study of new subpopulations, differentiation and dynamic evolution of cells more in-depth. The combination of scRNAseq technology and bioinformatics technology can effectively interpret the gene expression, splicing and other information obtained by sequencing, and construct the regulatory network based on these information, so as to analyze the role of synovial tissue in the occurrence and development of RA. ScRNA-seq technology still has great application space in the research of autoimmune diseases such as RA. In particular, the development of high-throughput single-cell RNA sequencing platform has carried out cell-to-cell difference analysis and exploration of potential mechanisms from multiple dimensions, and can predict the activity and radiological progress of RA from the characteristics of cell and molecular mechanism of synovial tissue, and predict the clinical efficacy of csDMARD treatment (111), which will bring greater breakthrough for RA treatment.

\section{AUTHOR CONTRIBUTIONS}

LC drafted the manuscript, drew illustrations, and discussed the content with the other authors. CW conceived the topic of the manuscript and revised the content of the manuscript. YW, RW, TD, and HX revised the manuscript. CG and XL also critically revised the content of the manuscript. All authors contributed to the article and approved the submitted version.

\section{FUNDING}

This work was supported by the National Natural Science Foundation of China (No. 81971543), National Natural Science Foundation of China (No. 8197061587), National Natural Science Foundation of China (No. 81471618), and Key Research and Development (R\&D) Projects of Shanxi Province (201803D31119). 


\section{REFERENCES}

1. Brondello JM, Djouad F, Jorgensen C. Where to Stand With Stromal Cells and Chronic Synovitis in Rheumatoid Arthritis? Cells (2019) 8(10):1257. doi: 10.3390/cells8101257

2. Croft AP, Campos J, Jansen K, Turner JD, Marshall J, Attar M, et al. Distinct Fibroblast Subsets Drive Inflammation and Damage in Arthritis. Nature (2019) 570(7760):246-51. doi: 10.1038/s41586-019-1263-7

3. Rana AK, Li Y, Dang Q, Yang F. Monocytes in Rheumatoid Arthritis: Circulating Precursors of Macrophages and Osteoclasts and, Their Heterogeneity and Plasticity Role in RA Pathogenesis. Int Immunopharmacol (2018) 65:348-59. doi: 10.1016/j.intimp.2018.10.016

4. Choy E. Understanding the Dynamics: Pathways Involved in the Pathogenesis of Rheumatoid Arthritis. Rheumatol (Oxford) (2012) 51 Suppl 5:v3-11. doi: 10.1093/rheumatology/kes113

5. Chen SJ, Lin GJ, Chen JW, Wang KC, Tien CH, Hu CF, et al. Immunopathogenic Mechanisms and Novel Immune-Modulated Therapies in Rheumatoid Arthritis. Int J Mol Sci (2019) 20(6):1332. doi: 10.3390/ijms20061332

6. Aletaha D, Smolen JS. Diagnosis and Management of Rheumatoid Arthritis: A Review. JAMA (2018) 320(13):1360-72. doi: 10.1001/jama.2018.13103

7. Ding T, Niu H, Zhao X, Gao C, Li X, Wang C, et al. T-Follicular Regulatory Cells: Potential Therapeutic Targets in Rheumatoid Arthritis. Front Immunol (2019) 10:2709. doi: 10.3389/fimmu.2019.02709

8. Kalisky T, Blainey P, Quake SR. Genomic Analysis at the Single-Cell Level. Annu Rev Genet (2011) 45:431-45. doi: 10.1146/annurev-genet-102209163607

9. Navin N, Kendall J, Troge J, Andrews P, Rodgers L, McIndoo J, et al. Tumour Evolution Inferred by Single-Cell Sequencing. Nature (2011) 472 (7341):90-4. doi: 10.1038/nature09807

10. Treutlein B, Lee QY, Camp JG, Mall M, Koh W, Shariati SA, et al. Dissecting Direct Reprogramming From Fibroblast to Neuron Using Single-Cell RNASeq. Nature (2016) 534(7607):391-5. doi: 10.1038/nature18323

11. Ji Q, Zheng Y, Zhang G, Hu Y, Fan X, Hou Y, et al. Single-Cell RNA-Seq Analysis Reveals the Progression of Human Osteoarthritis. Ann Rheum Dis (2019) 78(1):100-10. doi: 10.1136/annrheumdis-2017-212863

12. Gaublomme JT, Yosef N, Lee JY, Gertner RS, Yang LV, Wu C, et al. SingleCell Genomics Unveils Critical Regulators of Th17 Cell Pathogenicity. Cell (2015) 163(6):1400-12. doi: 10.1016/j.cell.2015.11.009

13. Ospelt C, Brentano F, Rengel Y, Stanczyk J, Kolling C, Tak PP, et al. Overexpression of Toll-Like Receptors 3 and 4 in Synovial Tissue From Patients With Early Rheumatoid Arthritis: Toll-Like Receptor Expression in Early and Longstanding Arthritis. Arthritis Rheum (2008) 58(12):3684-92. doi: 10.1002/art.24140

14. Collison J. Origins of Synovial Macrophages Revealed. Nat Rev Rheumatol (2019) 15(8):451. doi: 10.1038/s41584-019-0260-4

15. Tang F, Barbacioru C, Wang Y, Nordman E, Lee C, Xu N, et al. mRNA-Seq Whole-Transcriptome Analysis of a Single Cell. Nat Methods (2009) 6 (5):377-82. doi: 10.1038/nmeth.1315

16. Wang Y, Navin NE. Advances and Applications of Single-Cell Sequencing Technologies. Mol Cell (2015) 58(4):598-609. doi: 10.1016/ j.molcel.2015.05.005

17. Hedlund E, Deng Q. Single-Cell RNA Sequencing: Technical Advancements and Biological Applications. Mol Aspects Med (2017) 59:36-46. doi: 10.1016/ j.mam.2017.07.003

18. Lovett M. The Applications of Single-Cell Genomics. Hum Mol Genet (2013) 22(R1):R22-6. doi: 10.1093/hmg/ddt377

19. Asif Amin M, Fox DA, Ruth JH. Synovial Cellular and Molecular Markers in Rheumatoid Arthritis. Semin Immunopathol (2017) 39(4):385-93. doi: 10.1007/s00281-017-0631-3

20. Müller-Ladner U, Kriegsmann J, Franklin BN, Matsumoto S, Geiler T, Gay RE, et al. Synovial Fibroblasts of Patients With Rheumatoid Arthritis Attach to and Invade Normal Human Cartilage When Engrafted Into SCID Mice. Am J Pathol (1996) 149(5):1607-15. doi: 10.1111/j.1399-0039.1996. tb02701.x

21. Orr C, Vieira-Sousa E, Boyle DL, Buch MH, Buckley CD, Canete JD, et al. Synovial Tissue Research: A State-of-the-Art Review. Nat Rev Rheumatol (2017) 13(8):463-75. doi: 10.1038/nrrheum.2017.115
22. McInnes IB, Schett G. The Pathogenesis of Rheumatoid Arthritis. N Engl J Med (2011) 365(23):2205-19. doi: 10.1056/NEJMra1004965

23. Müller-Ladner U, Gay S. MMPs and Rheumatoid Synovial Fibroblasts: Siamese Twins in Joint Destruction? Ann Rheum Dis (2002) 61(11):957-9. doi: 10.1136/ard.61.11.957

24. Takayanagi H, Iizuka H, Juji T, Nakagawa T, Yamamoto A, Miyazaki T, et al. Involvement of Receptor Activator of Nuclear Factor KappaB Ligand/ Osteoclast Differentiation Factor in Osteoclastogenesis From Synoviocytes in Rheumatoid Arthritis. Arthritis Rheum (2000) 43(2):259-69. doi: 10.1002/ 1529-0131(200002)43:2<259::AID-ANR4>3.0.CO;2-W

25. Udalova IA, Mantovani A, Feldmann M. Macrophage Heterogeneity in the Context of Rheumatoid Arthritis. Nat Rev Rheumatol (2016) 12(8):472-85. doi: 10.1038/nrrheum.2016.91

26. Yang S, Ohe R, Aung NY, Kato T, Kabasawa T, Utsunomiya A, et al. Comparative Study of HO-1 Expressing Synovial Lining Cells Between RA and OA. Mod Rheumatol (2020) 1-8. doi: 10.1080/14397595.2019.1704976

27. Naylor AJ, Filer A, Buckley CD. The Role of Stromal Cells in the Persistence of Chronic Inflammation. Clin Exp Immunol (2013) 171(1):30-5. doi: 10.1111/j.1365-2249.2012.04634.x

28. Tang Y, Wang B, Sun X, Li H, Ouyang X, Wei J, et al. Rheumatoid Arthritis Fibroblast-Like Synoviocytes Co-Cultured With PBMC Increased Peripheral CD4(+) CXCR5(+) ICOS(+) T Cell Numbers. Clin Exp Immunol (2017) 190 (3):384-93. doi: 10.1111/cei.13025

29. Hirota K, Yoshitomi H, Hashimoto M, Maeda S, Teradaira S, Sugimoto N, et al. Preferential Recruitment of CCR6-Expressing Th17 Cells to Inflamed Joints via CCL20 in Rheumatoid Arthritis and Its Animal Model. J Exp Med (2007) 204(12):2803-12. doi: 10.1084/jem.20071397

30. Burger JA, Zvaifler NJ, Tsukada N, Firestein GS, Kipps TJ. Fibroblast-Like Synoviocytes Support B-Cell Pseudoemperipolesis via a Stromal CellDerived Factor-1- and CD106 (VCAM-1)-Dependent Mechanism. J Clin Invest (2001) 107(3):305-15. doi: 10.1172/JCI11092

31. McAllister E, Jellusova J. BAFF Signaling in B Cell Metabolism. Curr Opin Immunol (2021) 71:69-74. doi: 10.1016/j.coi.2021.05.011

32. Bombardieri M, Kam NW, Brentano F, Choi K, Filer A, Kyburz D, et al. A BAFF/APRIL-Dependent TLR3-Stimulated Pathway Enhances the Capacity of Rheumatoid Synovial Fibroblasts to Induce AID Expression and Ig ClassSwitching in B Cells. Ann Rheum Dis (2011) 70(10):1857-65. doi: 10.1136/ ard.2011.150219

33. Wang Y, Han CC, Cui D, Li Y, Ma Y, Wei W. Is Macrophage Polarization Important in Rheumatoid Arthritis? Int Immunopharmacol (2017) 50:34552. doi: 10.1016/j.intimp.2017.07.019

34. Koo J, Kim S, Jung WJ, Lee YE, Song GG, Kim KS, et al. Increased Lymphocyte Infiltration in Rheumatoid Arthritis Is Correlated With an Increase in LTi-Like Cells in Synovial Fluid. Immune Netw (2013) 13 (6):240-8. doi: 10.4110/in.2013.13.6.240

35. Alivernini S, MacDonald L, Elmesmari A, Finlay S, Tolusso B, Gigante MR, et al. Distinct Synovial Tissue Macrophage Subsets Regulate Inflammation and Remission in Rheumatoid Arthritis. Nat Med (2020) 26(8):1295-306. doi: 10.1038/s41591-020-0939-8

36. Gonzalo-Gil E, Galindo-Izquierdo M. Role of Transforming Growth FactorBeta (TGF) Beta in the Physiopathology of Rheumatoid Arthritis. Reumatol Clin (2014) 10(3):174-9. doi: 10.1016/j.reumae.2014.01.006

37. Perlman H, Bradley K, Liu H, Cole S, Shamiyeh E, Smith RC, et al. IL-6 and Matrix Metalloproteinase-1 Are Regulated by the Cyclin-Dependent Kinase Inhibitor P21 in Synovial Fibroblasts. J Immunol (2003) 170(2):838-45. doi: 10.4049/jimmunol.170.2.838

38. Onodera S, Kaneda K, Mizue Y, Koyama Y, Fujinaga M, Nishihira J, et al. Macrophage Migration Inhibitory Factor Up-Regulates Expression of Matrix Metalloproteinases in Synovial Fibroblasts of Rheumatoid Arthritis. J Biol Chem (2000) 275(1):444-50. doi: 10.1074/jbc.275.1.444

39. Onodera S, Nishihira J, Koyama Y, Majima T, Aoki Y, Ichiyama H, et al. Macrophage Migration Inhibitory Factor Up-Regulates the Expression of Interleukin-8 Messenger RNA in Synovial Fibroblasts of Rheumatoid Arthritis Patients: Common Transcriptional Regulatory Mechanism Between Interleukin-8 and Interleukin-1beta. Arthritis Rheum (2004) 50 (5):1437-47. doi: 10.1002/art.20190

40. Ospelt C. Synovial Fibroblasts in 2017. RMD Open (2017) 3(2):e000471. doi: 10.1136/rmdopen-2017-000471 
41. Zhang CW, Wu X, Liu D, Zhou W, Tan W, Fang YX, et al. Long non-Coding RNA PVT1 Knockdown Suppresses Fibroblast-Like Synoviocyte Inflammation and Induces Apoptosis in Rheumatoid Arthritis Through Demethylation of Sirt6. J Biol Eng (2019) 13:60. doi: 10.1186/s13036-0190184-1

42. Ai R, Hammaker D, Boyle DL, Morgan R, Walsh AM, Fan S, et al. JointSpecific DNA Methylation and Transcriptome Signatures in Rheumatoid Arthritis Identify Distinct Pathogenic Processes. Nat Commun (2016) 7:11849. doi: $10.1038 /$ ncomms 11849

43. Hammaker D, Nygaard G, Kuhs A, Ai R, Boyle DL, Wang W, et al. Joint Location-Specific JAK-STAT Signaling in Rheumatoid Arthritis FibroblastLike Synoviocytes. ACR Open Rheumatol (2019) 1(10):640-8. doi: 10.1002/ acr2.11093

44. Croft AP, Naylor AJ, Marshall JL, Hardie DL, Zimmermann B, Turner J, et al. Rheumatoid Synovial Fibroblasts Differentiate Into Distinct Subsets in the Presence of Cytokines and Cartilage. Arthritis Res Ther (2016) 18(1):270. doi: 10.1186/s13075-016-1156-1

45. Frank-Bertoncelj M, Trenkmann M, Klein K, Karouzakis E, Rehrauer H, Bratus A, et al. Epigenetically-Driven Anatomical Diversity of Synovial Fibroblasts Guides Joint-Specific Fibroblast Functions. Nat Commun (2017) 8:14852. doi: 10.1038/ncomms14852

46. Kiener HP, Niederreiter B, Lee DM, Jimenez-Boj E, Smolen JS, Brenner MB. Cadherin 11 Promotes Invasive Behavior of Fibroblast-Like Synoviocytes. Arthritis Rheum (2009) 60(5):1305-10. doi: 10.1002/art.24453

47. Kiener HP, Lee DM, Agarwal SK, Brenner MB. Cadherin-11 Induces Rheumatoid Arthritis Fibroblast-Like Synoviocytes to Form Lining Layers In Vitro. Am J Pathol (2006) 168(5):1486-99. doi: 10.2353/ajpath.2006. 050999

48. Karpus ON, Kiener HP, Niederreiter B, Yilmaz-Elis AS, van der Kaa J, Ramaglia V, et al. CD55 Deposited on Synovial Collagen Fibers Protects From Immune Complex-Mediated Arthritis. Arthritis Res Ther (2015) 17 (1):6. doi: 10.1186/s13075-015-0518-4

49. Maia M, de Vriese A, Janssens T, Moons M, van Landuyt K, Tavernier J, et al. CD248 and its Cytoplasmic Domain: A Therapeutic Target for Arthritis. Arthritis Rheum (2010) 62(12):3595-606. doi: 10.1002/art.27701

50. Kraman M, Bambrough PJ, Arnold JN, Roberts EW, Magiera L, Jones JO, et al. Suppression of Antitumor Immunity by Stromal Cells Expressing Fibroblast Activation Protein-Alpha. Science (2010) 330(6005):827-30. doi: $10.1126 /$ science. 1195300

51. Lax S, Hou TZ, Jenkinson E, Salmon M, MacFadyen JR, Isacke CM, et al. CD248/Endosialin is Dynamically Expressed on a Subset of Stromal Cells During Lymphoid Tissue Development, Splenic Remodeling and Repair. FEBS Lett (2007) 581(18):3550-6. doi: 10.1016/j.febslet.2007.06.063

52. Saalbach A, Kraft R, Herrmann K, Haustein UF, Anderegg U. The Monoclonal Antibody AS02 Recognizes a Protein on Human Fibroblasts Being Highly Homologous to Thy-1. Arch Dermatol Res (1998) 290(7):3606. doi: $10.1007 / \mathrm{s} 004030050318$

53. Stephenson W, Donlin LT, Butler A, Rozo C, Bracken B, Rashidfarrokhi A, et al. Single-Cell RNA-Seq of Rheumatoid Arthritis Synovial Tissue Using Low-Cost Microfluidic Instrumentation. Nat Commun (2018) 9(1):791. doi: 10.1038/s41467-017-02659-x

54. Xue J, Chen J, Shen Q, Chan D, Li J, Tanguay AP, et al. Addition of High Molecular Weight Hyaluronic Acid to Fibroblast-Like Stromal Cells Modulates Endogenous Hyaluronic Acid Metabolism and Enhances Proteolytic Processing and Secretion of Versican. Cells (2020) 9(7):1681. doi: $10.3390 /$ cells 9071681

55. Zhang F, Wei K, Slowikowski K, Fonseka CY, Rao DA, Kelly S, et al. Defining Inflammatory Cell States in Rheumatoid Arthritis Joint Synovial Tissues by Integrating Single-Cell Transcriptomics and Mass Cytometry. Nat Immunol (2019) 20(7):928-42. doi: 10.1101/351130

56. Alquraini A, Garguilo S, Zhang L, Schmidt T, Jay GE, lsaid KA. The Interaction of Lubricin/Proteoglycan-4 (PRG4) With Toll-Like Receptor 2: An Anti-Inflammatory Role of PRG4 in Synovial Fluids From Patients With Osteoarthritis. Osteoarthritis Cartilage (2016) 24:S499-500. doi: 10.1016/ j.joca.2016.01.915

57. Oguchi T, Ishiguro N. Differential Stimulation of Three Forms of Hyaluronan Synthase by TGF-Beta, IL-1beta, and TNF-Alpha. Connect Tissue Res (2004) 45(4-5):197-205. doi: 10.1080/03008200490523031
58. Lee WS, Kato M, Sugawara E, Kono M, Kudo Y, Kono M, et al. Optineurin in Synovial Fibroblasts Plays a Protective Role Against Joint Destructions in Rheumatoid Arthritis. Arthritis Rheumatol (2020) 72(9):1493-504. doi: $10.1002 /$ art. 41290

59. Mizoguchi F, Slowikowski K, Wei K, Marshall JL, Rao DA, Chang SK, et al. Functionally Distinct Disease-Associated Fibroblast Subsets in Rheumatoid Arthritis. Nat Commun (2018) 9(1):789. doi: 10.1038/s41467-018-02892-y

60. Suzuki T, Takakubo Y, Oki H, Liu X, Honma R, Naganuma Y, et al. Immunohistochemical Analysis of Inflammatory Rheumatoid Synovial Tissues Using Anti-Human Podoplanin Monoclonal Antibody Panel. Monoclon Antib Immunodiagn Immunother (2018) 37(1):12-9. doi: 10.1089/mab.2017.0047

61. Wicki A, Lehembre F, Wick N, Hantusch B, Kerjaschki D, Christofori G, et al. Tumor Invasion in the Absence of Epithelial-Mesenchymal Transition: Podoplanin-Mediated Remodeling of the Actin Cytoskeleton. Cancer Cell (2006) 9(4):261-72. doi: 10.1016/j.ccr.2006.03.010

62. Park YE, Woo YJ, Park SH, Moon YM, Oh HJ, Kim JI, et al. IL-17 Increases Cadherin-11 Expression in a Model of Autoimmune Experimental Arthritis and in Rheumatoid Arthritis. Immunol Lett (2011) 140(1-2):97-103. doi: 10.1016/j.imlet.2011.07.003

63. Yu Z, Xu H, Wang H, Wang Y. Foxcl Promotes the Proliferation of Fibroblast-Like Synoviocytes in Rheumatoid Arthritis via PI3K/AKT Signalling Pathway. Tissue Cell (2018) 53:15-22. doi: 10.1016/ j.tice.2018.05.011

64. Hirohata S, Yanagida T, Nagai T, Sawada T, Nakamura H, Yoshino S, et al. Induction of Fibroblast-Like Cells From CD34(+) Progenitor Cells of the Bone Marrow in Rheumatoid Arthritis. J Leukoc Biol (2001) 70(3):413-21. doi: $10.1189 / \mathrm{jlb} .70 .3 .413$

65. Johnnidis JB, Camargo FD. Isolation and Functional Characterization of Side Population Stem Cells. Methods Mol Biol (2008) 430:183-93. doi: 10.1007/978-1-59745-182-6_13

66. Jo $\mathrm{CH}$, Ahn HJ, Kim HJ, Seong SC, Lee MC. Surface Characterization and Chondrogenic Differentiation of Mesenchymal Stromal Cells Derived From Synovium. Cytotherapy (2007) 9(4):316-27. doi: 10.1080/ 14653240701291620

67. Vukicevic S, Grgurevic L. BMP-6 and Mesenchymal Stem Cell Differentiation. Cytokine Growth Factor Rev (2009) 20(5-6):441-8. doi: 10.1016/j.cytogfr.2009.10.020

68. Chiu YG, Ritchlin CT. Denosumab: Targeting the RANKL Pathway to Treat Rheumatoid Arthritis. Expert Opin Biol Ther (2017) 17(1):119-28. doi: 10.1080/14712598.2017.1263614

69. Komatsu N, Okamoto K, Sawa S, Nakashima T, Oh-hora M, Kodama T, et al. Pathogenic Conversion of Foxp3+ T Cells Into TH17 Cells in Autoimmune Arthritis. Nat Med (2014) 20(1):62-8. doi: 10.1038/nm.3432

70. Lee A, Qiao Y, Grigoriev G, Chen J, Park-Min KH, Park SH, et al. Tumor Necrosis Factor $\alpha$ Induces Sustained Signaling and a Prolonged and Unremitting Inflammatory Response in Rheumatoid Arthritis Synovial Fibroblasts. Arthritis Rheum (2013) 65(4):928-38. doi: 10.1002/art.37853

71. Badolato R, Oppenheim JJ. Role of Cytokines, Acute-Phase Proteins, and Chemokines in the Progression of Rheumatoid Arthritis. Semin Arthritis Rheum (1996) 26(2):526-38. doi: 10.1016/S0049-0172(96)80041-2

72. Kimura A, Kishimoto T. IL-6: Regulator of Treg/Th17 Balance. Eur J Immunol (2010) 40(7):1830-5. doi: 10.1002/eji.201040391

73. Nurieva RI, Chung Y, Hwang D, Yang XO, Kang HS, Ma L, et al. Generation of T Follicular Helper Cells is Mediated by Interleukin-21 But Independent of T Helper 1, 2, or 17 Cell Lineages. Immunity (2008) 29(1):138-49. doi: 10.1016/j.immuni.2008.05.009

74. Pickens SR, Chamberlain ND, Volin MV, Gonzalez M, Pope RM, Mandelin AM, et al. Anti-CXCL5 Therapy Ameliorates IL-17-Induced Arthritis by Decreasing Joint Vascularization. Angiogenesis (2011) 14(4):443-55. doi: 10.1007/s10456-011-9227-z

75. Pickens SR, Volin MV, Mandelin AM, Kolls JK, Pope RM, Shahrara S, et al. IL17 Contributes to Angiogenesis in Rheumatoid Arthritis. J Immunol (2010) 184 (6):3233-41. doi: 10.4049/jimmunol.0903271

76. Karnowski A, Chevrier S, Belz GT, Mount A, Emslie D, D'Costa K, et al. B and T Cells Collaborate in Antiviral Responses via IL-6, IL-21, and Transcriptional Activator and Coactivator, Oct2 and OBF-1. J Exp Med (2012) 209(11):2049-64. doi: 10.1084/jem.20111504 
77. Liu R, Zhao P, Zhang Q, Che N, Xu L, Qian J, et al. Adiponectin Promotes Fibroblast-Like Synoviocytes Producing IL-6 to Enhance T Follicular Helper Cells Response in Rheumatoid Arthritis. Clin Exp Rheumatol (2020) 38 (1):11-8.

78. Seidel MF, Koch FW, Vetter H. Macrophage-Like Synoviocytes Display Phenotypic Polymorphisms in a Serum-Free Tissue-Culture Medium. Rheumatol Int (2006) 26(3):244-51. doi: 10.1007/s00296-004-0545-y

79. Snelling SJ, Davidson RK, Swingler TE, Le LT, Barter MJ, Culley KL, et al. Dickkopf-3 Is Upregulated in Osteoarthritis and Has a Chondroprotective Role. Osteoarthritis Cartilage (2016) 24(5):883-91. doi: 10.1016/ j.joca.2015.11.021

80. Turner JD, Filer A. The Role of the Synovial Fibroblast in Rheumatoid Arthritis Pathogenesis. Curr Opin Rheumatol (2015) 27(2):175-82. doi: 10.1097/BOR.0000000000000148

81. Wei K, Korsunsky I, Marshall JL, Gao A, Watts GFM, Major T, et al. Notch Signalling Drives Synovial Fibroblast Identity and Arthritis Pathology. Nature (2020) 582(7811):259-64. doi: 10.1038/s41586-020-2222-Z

82. Montero-Melendez T, Nagano A, Chelala C, Filer A, Buckley CD, Perretti M. Therapeutic Senescence via GPCR Activation in Synovial Fibroblasts Facilitates Resolution of Arthritis. Nat Commun (2020) 11(1):745. doi: 10.1038/s41467-020-14421-x

83. Milner JM, Patel A, Rowan AD. Emerging Roles of Serine Proteinases in Tissue Turnover in Arthritis. Arthritis Rheum (2008) 58(12):3644-56. doi: 10.1002/art.24046

84. Wäldele S, Koers-Wunrau C, Beckmann D, Korb-Pap A, Wehmeyer C, Pap T, et al. Deficiency of Fibroblast Activation Protein Alpha Ameliorates Cartilage Destruction in Inflammatory Destructive Arthritis. Arthritis Res Ther (2015) 17(1):12. doi: 10.1186/s13075-015-0524-6

85. Choi IY, Karpus ON, Turner JD, Hardie D, Marshall JL, de Hair MJH, et al. Stromal Cell Markers are Differentially Expressed in the Synovial Tissue of Patients With Early Arthritis. PloS One (2017) 12(8):e0182751. doi: 10.1371/ journal.pone.0182751

86. Dennis G Jr, Holweg CT, Kummerfeld SK, Choy DF, Setiadi AF, Hackney JA, et al. Synovial Phenotypes in Rheumatoid Arthritis Correlate With Response to Biologic Therapeutics. Arthritis Res Ther (2014) 16(2):R90. doi: 10.1186/ $\operatorname{ar} 4555$

87. Bombardieri M, Lewis M, Pitzalis C. Ectopic Lymphoid Neogenesis in Rheumatic Autoimmune Diseases. Nat Rev Rheumatol (2017) 13(3):14154. doi: 10.1038/nrrheum.2016.217

88. Rivellese F, Humby F, Bugatti S, Fossati-Jimack L, Rizvi H, Lucchesi D, et al. B Cell Synovitis and Clinical Phenotypes in Rheumatoid Arthritis: Relationship to Disease Stages and Drug Exposure. Arthritis Rheumatol (2020) 72(5):714-25. doi: 10.1002/art.41184

89. Huang W, Zhang L, Cheng C, Shan W, Ma R, Yin Z, et al. Parallel Comparison of Fibroblast-Like Synoviocytes From the Surgically Removed Hyperplastic Synovial Tissues of Rheumatoid Arthritis and Osteoarthritis Patients. BMC Musculoskelet Disord (2019) 20(1):591. doi: 10.1186/s12891019-2977-2

90. Nygaard G, Firestein GS. Restoring Synovial Homeostasis in Rheumatoid Arthritis by Targeting Fibroblast-Like Synoviocytes. Nat Rev Rheumatol (2020) 16(6):316-33. doi: 10.1038/s41584-020-0413-5

91. Filer A. The Fibroblast as a Therapeutic Target in Rheumatoid Arthritis. Curr Opin Pharmacol (2013) 13(3):413-9. doi: 10.1016/j.coph.2013.02.006

92. Hamilton JA, Tak PP. The Dynamics of Macrophage Lineage Populations in Inflammatory and Autoimmune Diseases. Arthritis Rheum (2009) 60 (5):1210-21. doi: 10.1002/art.24505

93. Epelman S, Lavine KJ, Randolph GJ. Origin and Functions of Tissue Macrophages. Immunity (2014) 41(1):21-35. doi: 10.1016/j.immuni.2014.06.013

94. Tu J, Wang X, Gong X, Hong W, Han D, Fang Y, et al. Synovial Macrophages in Rheumatoid Arthritis: The Past, Present, and Future. Mediators Inflammation (2020) 2020:1583647. doi: 10.1155/2020/1583647

95. Bain CC, Bravo-Blas A, Scott CL, Perdiguero EG, Geissmann F, Henri S, et al. Constant Replenishment From Circulating Monocytes Maintains the Macrophage Pool in the Intestine of Adult Mice. Nat Immunol (2014) 15 (10):929-37. doi: 10.1038/ni.2967

96. Yona S, Kim KW, Wolf Y, Mildner A, Varol D, Breker M, et al. Fate Mapping Reveals Origins and Dynamics of Monocytes and Tissue
Macrophages Under Homeostasis. Immunity (2013) 38(1):79-91. doi: 10.1016/j.immuni.2012.12.001

97. Bain CC, Hawley CA, Garner H, Scott CL, Schridde A, Steers NJ, et al. LongLived Self-Renewing Bone Marrow-Derived Macrophages Displace EmbryoDerived Cells to Inhabit Adult Serous Cavities. Nat Commun (2016) 7: ncomms11852. doi: $10.1038 /$ ncomms 11852

98. Hashimoto D, Chow A, Noizat C, Teo P, Beasley MB, Leboeuf M, et al. TissueResident Macrophages Self-Maintain Locally Throughout Adult Life With Minimal Contribution From Circulating Monocytes. Immunity (2013) 38 (4):792-804. doi: 10.1016/j.immuni.2013.04.004

99. Sica A, Mantovani A. Macrophage Plasticity and Polarization: In Vivo Veritas. J Clin Invest (2012) 122(3):787-95. doi: 10.1172/JCI59643

100. Ginhoux F, Schultze JL, Murray PJ, Ochando J, Biswas SK. New Insights Into the Multidimensional Concept of Macrophage Ontogeny, Activation and Function. Nat Immunol (2016) 17(1):34-40. doi: 10.1038/ni.3324

101. Mantovani A, Sica A, Sozzani S, Allavena P, Vecchi A, Locati M. The Chemokine System in Diverse Forms of Macrophage Activation and Polarization. Trends Immunol (2004) 25(12):677-86. doi: 10.1016/ j.it.2004.09.015

102. Bashir S, Sharma Y, Elahi A, Khan F. Macrophage Polarization: The Link Between Inflammation and Related Diseases. Inflammation Res (2016) 65 (1):1-11. doi: 10.1007/s00011-015-0874-1

103. Krausgruber T, Blazek K, Smallie T, Alzabin S, Lockstone H, Sahgal N, et al. IRF5 Promotes Inflammatory Macrophage Polarization and TH1-TH17 Responses. Nat Immunol (2011) 12(3):231-8. doi: 10.1038/ni.1990

104. Misharin AV, Cuda CM, Saber R, Turner JD, Gierut AK, Haines GK, et al. Nonclassical Ly6C(-) Monocytes Drive the Development of Inflammatory Arthritis in Mice. Cell Rep (2014) 9(2):591-604. doi: 10.1016/j.celrep. 2014.09.032

105. Donlin LT, Jayatilleke A, Giannopoulou EG, Kalliolias GD, Ivashkiv LB. Modulation of TNF-Induced Macrophage Polarization by Synovial Fibroblasts. J Immunol (2014) 193(5):2373-83. doi: 10.4049/jimmunol.1400486

106. Zhang H, Lin C, Zeng C, Wang Z, Wang H, Lu J, et al. Synovial Macrophage M1 Polarisation Exacerbates Experimental Osteoarthritis Partially Through R-Spondin-2. Ann Rheum Dis (2018) 77(10):1524-34. doi: 10.1136/ annrheumdis-2018-213450

107. Tu J, Hong W, Guo Y, Zhang P, Fang Y, Wang X, et al. Ontogeny of Synovial Macrophages and the Roles of Synovial Macrophages From Different Origins in Arthritis. Front Immunol (2019) 10:1146. doi: 10.3389/fimmu.2019.01146

108. Kuo D, Ding J, Cohn IS, Zhang F, Wei K, Rao DA, et al. HBEGF(+) Macrophages in Rheumatoid Arthritis Induce Fibroblast Invasiveness. Sci Transl Med (2019) 11:491. doi: 10.1126/scitranslmed.aau8587

109. Stubbington MJT, Rozenblatt-Rosen O, Regev A, Teichmann SA. Single-Cell Transcriptomics to Explore the Immune System in Health and Disease. Science (2017) 358(6359):58-63. doi: 10.1126/science.aan6828

110. Culemann S, Grüneboom A, Nicolás-Ávila J, Weidner D, Lämmle KF, Rothe $\mathrm{T}$, et al. Locally Renewing Resident Synovial Macrophages Provide a Protective Barrier for the Joint. Nature (2019) 572(7771):670-5. doi: 10.1038/s41586-019-1471-1

111. Humby F, Lewis M, Ramamoorthi N, Hackney JA, Barnes MR, Bombardieri M, et al. Synovial Cellular and Molecular Signatures Stratify Clinical Response to csDMARD Therapy and Predict Radiographic Progression in Early Rheumatoid Arthritis Patients. Ann Rheum Dis (2019) 78(6):761-72. doi: 10.1136/annrheumdis-2018-214539

Conflict of Interest: The authors declare that the research was conducted in the absence of any commercial or financial relationships that could be construed as a potential conflict of interest.

Copyright (c) 2021 Cheng, Wang, Wu, Ding, Xue, Gao, Li and Wang. This is an openaccess article distributed under the terms of the Creative Commons Attribution License (CC BY). The use, distribution or reproduction in other forums is permitted, provided the original author(s) and the copyright owner(s) are credited and that the original publication in this journal is cited, in accordance with accepted academic practice. No use, distribution or reproduction is permitted which does not comply with these terms. 